\title{
ON GENERALIZATIONS OF FATOU'S THEOREM FOR THE INTEGRALS WITH GENERAL KERNELS
}

\author{
G. A. KARAGULYAN AND M. H. SAFARYAN
}

\begin{abstract}
We define $\lambda(r)$-convergence, which is a generalization of nontangential convergence in the unit disc. We prove Fatou-type theorems on almost everywhere nontangential convergence of Poisson-Stiltjes integrals for general kernels $\left\{\varphi_{r}\right\}$, forming an approximation of identity. We prove that the bound

$$
\limsup _{r \rightarrow 1} \lambda(r)\left\|\varphi_{r}\right\|_{\infty}<\infty
$$

is necessary and sufficient for almost everywhere $\lambda(r)$-convergence of the integrals

$$
\int_{\mathbb{T}} \varphi_{r}(t-x) d \mu(t)
$$
\end{abstract}

\section{INTRODUCTION}

In his famous paper Fatou [5] proved

Theorem A (Fatou, 1906). Any bounded analytic function on the unit disc $D=$ $\{z \in \mathbb{C}:|z|<1\}$ has nontangential limit for almost all boundary points.

Theorem B (Fatou, 1906). If a function of bounded variation $\mu(t)$ is differentiable at $x_{0} \in \mathbb{T}$, then the Poisson integral

$$
\mathcal{P}_{r}(x, d \mu)=\frac{1}{2 \pi} \int_{\mathbb{T}} \frac{1-r^{2}}{1-2 r \cos (x-t)+r^{2}} d \mu(t)
$$

converges non-tangentially to $\mu^{\prime}\left(x_{0}\right)$ as $r \rightarrow 1$.

These are two fundamental theorems, having many applications in different mathematical theories (analytic functions, Hardy spaces, harmonic analysis, differential equations and etc ). J. Littlewood [7] made an important complement to these theorems, proving essentiality of nontangential approach in Fatou's theorems.

Theorem C (Littlewood, 1927). Let $\gamma \subset \bar{D}$ be an arbitrary curve, internally tangent at $z=1$ and no having other common point with $|z|=1$. Let $\gamma_{x}$ be the rotation of $\gamma$ about the origin by $e^{i x}$. Then there exists a bounded analytic function $f(z), z \in D$, which does not have boundary limit along $\gamma_{x}$ for almost every $e^{i x}$.

There are various generalization of these theorems in different aspects. Lohwater and Piranian [8] improved Littlewood's theorem, replacing almost everywhere divergence to everywhere. H. Aikawa in [1, 2] extended Littlewood's theorem for harmonic functions. Almost everywhere convergence over some semi-tangential regions investigated by Nagel and Stein [9, Di Biase [3], Di Biase-Stokolos-Svensson-Weiss [4. P. Sjögren ([14, [15], [16]), J.-O. Rönning ([10], [11, [12]), I. N. Katkovskaya

1991 Mathematics Subject Classification. Primary 42B25; Secondary 32A40.

Key words and phrases. Fatou theorem, Littlewood theorem, harmonic functions. 
and V. G. Krotov ([6]) obtained some tangential convergence properties for the square root Poisson integral. Unfortunately, we are not able to talk about the details of these investigations within this paper. Some of them will be discussed in the last section.

We define $\lambda(r)$-convergence, which is a generalization of nontangential convergence in the unit disc. Let $\lambda(r):(0,1) \rightarrow \mathbb{R}_{+}$be a function with $\lambda(r) \searrow 0$ as $r \rightarrow 1$. For a given $x \in \mathbb{T}$ we define $\lambda(r, x)$ to be the interval $[x-\lambda(r), x+\lambda(r)]$. If $\lambda(r) \geq \pi$ we assume that $\lambda(r, x)=\mathbb{T}$. Let $F_{r}(x)$ be a family of functions from $L^{1}(\mathbb{T})$, where $r$ varies in $(0,1)$. We say $F_{r}(x)$ is $\lambda(r)$-convergent at a point $x \in \mathbb{T}$ to a value $A$, if

$$
\lim _{r \rightarrow 1} \sup _{\theta \in \lambda(r, x)}\left|F_{r}(\theta)-A\right|=0 .
$$

Otherwise this relation will be denoted by

$$
\lim _{\substack{r \rightarrow 1 \\ \theta \in \lambda(r, x)}} F_{r}(\theta)=A
$$

It is clear, that the non-tangential convergence in the unit disc is the case of $\lambda(r)=$ $c(1-r)$.

Given function of bounded variation $\mu(t)$ defines Borel measure on $\mathbb{T}$. We consider the family of integrals

$$
\Phi_{r}(x, d \mu)=\int_{\mathbb{T}} \varphi_{r}(x-t) d \mu(t), \quad 0<r<1,
$$

where kernels $\varphi_{r}(x) \in L^{\infty}(\mathbb{T})$ form an approximative of identity (AI), that is

1. $\int_{\mathbb{T}} \varphi_{r}(t) d t \rightarrow 1$ as $r \rightarrow 1$,

2. $\varphi_{r}^{*}(x)=\sup _{|x| \leq|t| \leq \pi}\left|\varphi_{r}(t)\right| \rightarrow 0$ as $r \rightarrow 1,0<|x|<\pi$,

3. $\sup _{0<r<1} \int_{\mathbb{T}} \varphi_{r}^{*}(x)<\infty$.

If $\mu(t)$ is absolute continuous and $d \mu(t)=f(t) d t, f \in L^{1}(\mathbb{T})$, then (1.1) will be denoted by $\Phi_{r}(x, f)$. We shall prove that the condition

$$
\limsup _{r \rightarrow 1} \lambda(r)\left\|\varphi_{r}\right\|_{\infty}<\infty
$$

is necessary and sufficient for almost everywhere $\lambda(r)$-convergence of the integrals $\Phi_{r}(x, d \mu)$ as well as $\Phi_{r}(x, f), f \in L^{1}(\mathbb{T})$. Moreover we prove that convergence holds at any point where $\mu(t)$ is differentiable. An analogous necessary and sufficient condition will be established also for almost everywhere $\lambda(r)$-convergence of $\Phi_{r}(x, f)$ if $f \in L^{\infty}(\mathbb{T})$, and this condition looks like

$$
\lim _{\tau \rightarrow 0}\left(\limsup _{\delta \rightarrow 0} \sup _{\tau<r<1} \int_{-\delta \lambda(r)}^{\delta \lambda(r)} \varphi_{r}(t) d t\right)=0 .
$$

If $\varphi_{r}$ coincides with the Poisson kernel, then $\left\|\varphi_{r}\right\|_{\infty}=O(1 /(1-r))$ and from this results we deduce Fatou's theorems. Other consequences will be discussed in the last section. 


\section{FAtou Type theOREMS: THE CASE OF BOUNDED MESURES}

We denote by BV $(\mathbb{T})$ the functions of bounded variation on $\mathbb{T}$. We say that the given approximation of identity $\left\{\varphi_{r}(x)\right\}$ is regular if each $\varphi_{r}(x)$ is positive, decreasing on $[0, \pi]$ and increasing on $[-\pi, 0]$. In this case the property 3$)$ is unnecessary, because it will immediately follows from 1 ).

Theorem 2.1. Let $\left\{\varphi_{r}\right\}$ be a regular $A I$ and $\lambda(r)$ satisfies the condition

$$
\limsup _{r \rightarrow 1} \lambda(r)\left\|\varphi_{r}\right\|_{\infty}<\infty
$$

If $\mu(t) \in \mathrm{BV}(\mathbb{T})$ is differentiable at $x_{0}$, then

$$
\lim _{\substack{r \rightarrow 1 \\ x \in \lambda\left(r, x_{0}\right)}} \Phi_{r}(x, d \mu)=\mu^{\prime}\left(x_{0}\right) .
$$

An analogous theorem holds as well in the non-regular case of kernels, but at this time the points where (1.1) converges satisfy strong differentiability condition. We say a function of bounded variation is strong differentiable at $x_{0} \in \mathbb{T}$, if the there exist a number $c$ such that the variation of the function $\mu(x)-c x$ has zero derivative at $x_{0}$. If $d \mu(t)=f(t) d t$ this property means that $x_{0}$ is Lebesgue point for $f(x)$, that is

$$
\lim _{h \rightarrow 0} \frac{1}{2 h} \int_{-h}^{h}\left|f(x)-f\left(x_{0}\right)\right| d x=0 .
$$

It is well-known that strong differentiability at $x_{0}$ implies the existence of $\mu^{\prime}\left(x_{0}\right)$, and any function of bounded variation is strong differentiable almost everywhere.

Theorem 2.2. Let $\left\{\varphi_{r}\right\}$ be an arbitrary $A I$ and $\lambda(r)$ satisfies the condition (2.1). If $\mu(t) \in \mathrm{BV}(\mathbb{T})$ is strong differentiable at $x_{0} \in \mathbb{T}$, then

$$
\lim _{\substack{r \rightarrow 1 \\ x \in \lambda\left(r, x_{0}\right)}} \Phi_{r}(x, d \mu)=\mu^{\prime}\left(x_{0}\right) .
$$

The following theorem implies the sharpness of the condition (2.1) in Theorem 2.1 and Theorem 2.2

Theorem 2.3. If $\left\{\varphi_{r}(x) \geq 0\right\}$ is an $A I$ and the function $\lambda(r)$ satisfies the condition

$$
\limsup _{r \rightarrow 1} \lambda(r)\left\|\varphi_{r}\right\|_{\infty}=\infty
$$

then there exist a positive function $f \in L^{1}(\mathbb{T})$ such that

$$
\limsup _{\substack{r \rightarrow 1 \\ y \in \lambda(r, x)}} \Phi_{r}(y, f)=\infty
$$

for all $x \in \mathbb{T}$.

The following lemma plays significant role in the proofs of Theorem 2.1 and Theorem 2.2.

Lemma 2.4. Let a positive function $\varphi(t) \in L^{\infty}(\mathbb{T})$ is decreasing on $[0, \pi]$ and increasing on $[-\pi, 0]$. Then for any numbers $\varepsilon \in(0,1)$ and $\theta \in(-\pi, \pi)$ there exist 
a finite family of intervals $I_{j} \subset \mathbb{T}, j=1,2, \ldots, n$, containing 0 in their closures $\bar{I}_{j}$, and numbers $\varepsilon_{j}= \pm \varepsilon$ such that

$$
\begin{aligned}
& \left|I_{j}\right| \leq 2 \sup \{|t|: \varphi(t) \geq \varepsilon\}, \quad j=1,2, \ldots, n, \\
& \sum_{j=1}^{n}\left|I_{j}\right|<10 \varepsilon^{-1} \max \left\{1,|\theta| \cdot\|\varphi\|_{\infty},\|\varphi\|_{1}\right\}, \\
& \left|\varphi(x-\theta)-\sum_{j=1}^{n} \varepsilon_{j} \mathbb{I}_{I_{j}}(x)\right| \leq \varepsilon .
\end{aligned}
$$

Proof. Denote

$$
\begin{aligned}
& y_{k}=\sup \{t>0: \varphi(t) \geq \varepsilon k\}, \\
& x_{k}=\sup \{t>0: \varphi(-t) \geq \varepsilon k\}, \quad k=1,2, \ldots, l=\left[\frac{\|\varphi\|_{\infty}}{\varepsilon}\right] .
\end{aligned}
$$

Then we obviously have

$$
\begin{aligned}
& y_{0}=\pi, \quad 0 \leq y_{l} \leq y_{l-1} \leq \ldots \leq y_{1} \leq \sup \{|t|: \varphi(t) \geq \varepsilon\} \\
& x_{0}=\pi, \quad 0 \leq x_{l} \leq x_{l-1} \leq \ldots \leq x_{1} \leq \sup \{|t|: \varphi(t) \geq \varepsilon\}, \\
& \left|\varphi(x-\theta)-\varepsilon \sum_{k=1}^{l} \mathbb{I}_{\left(\theta-x_{k}, \theta+y_{k}\right)}(x)\right| \leq \varepsilon .
\end{aligned}
$$

Without loss of generality we can suppose $0 \leq \theta<\pi$. Then we denote

$$
k_{0}=\max \left\{k: 0 \leq k \leq l, \theta-x_{k} \leq 0\right\} .
$$

We define the desired intervals $I_{j}, j=1,2, \ldots, n=2 l-k_{0}$, by

$$
I_{j}=\left\{\begin{array}{lll}
\left(\theta-x_{j}, \theta+y_{j}\right) & \text { if } \quad j \leq k_{0}, \\
\left(0, \theta+y_{j}\right) & \text { if } \quad k_{0}<j \leq l, \\
\left(0, \theta-x_{j-l+k_{0}}\right] & \text { if } \quad l<j \leq n=2 l-k_{0} .
\end{array}\right.
$$

Using the equality

$$
\begin{aligned}
& \mathbb{I}_{\left(\theta-x_{k}, \theta+y_{k}\right)}(x) \\
& \quad=\mathbb{I}_{\left(0, \theta+y_{k}\right)}(x)-\mathbb{I}_{\left(0, \theta-x_{k}\right]}(x)=\mathbb{I}_{I_{k}}(x)-\mathbb{I}_{I_{k+l-k_{0}}}(x), \quad k_{0}<k \leq l,
\end{aligned}
$$

we get

$$
\varepsilon \sum_{k=1}^{l} \mathbb{I}_{\left(\theta-x_{k}, \theta+y_{k}\right)}(x)=\sum_{j=1}^{n} \varepsilon_{j} \mathbb{I}_{I_{j}}(x),
$$

where

$$
\varepsilon_{j}=\left\{\begin{array}{rll}
\varepsilon & \text { if } & 1 \leq j \leq l, \\
-\varepsilon & \text { if } & l<j \leq n .
\end{array}\right.
$$

We note that $\varepsilon_{j}=-\varepsilon$ in the case when $I_{j}$ coincides with one of the intervals $\left(0, \theta-x_{k}\right], k_{0}<k \leq l$. Hence we have

$$
\sum_{j=l+1}^{n}\left|I_{j}\right|=\sum_{k=k_{0}+1}^{l}\left(\theta-x_{k}\right) \leq l \cdot \theta \leq \frac{\theta\|\varphi\|_{\infty}}{\varepsilon} .
$$


From (2.6) and (2.7) we get

$$
\left|\varphi(x-\theta)-\sum_{j=1}^{n} \varepsilon_{j} \mathbb{I}_{I_{j}}(x)\right| \leq \varepsilon
$$

and therefore by (2.8) we obtain

$$
\left|\int_{\mathbb{T}} \varphi(t) d t-\varepsilon \sum_{j=1}^{l}\right| I_{j}\left|+\varepsilon \sum_{j=l+1}^{n}\right| I_{j}|| \leq 2 \pi \varepsilon<2 \pi .
$$

This and (2.9) imply

$$
\varepsilon \sum_{j=1}^{n}\left|I_{j}\right| \leq 2 \varepsilon \sum_{j=l+1}^{n}\left|I_{j}\right|+\|\varphi\|_{1}+2 \pi \leq 2 \theta\|\varphi\|_{\infty}+\|\varphi\|_{1}+2 \pi,
$$

which together with (2.4), (2.5) and (2.10) completes the proof of lemma.

Proof of Theorem 2.1. Without loss of generality we may assume that $x_{0}=0$ and $\mu^{\prime}\left(x_{0}\right)=0$. We fix a function $\theta(r):(0,1) \rightarrow \mathbb{R}$ with $|\theta(r)| \leq \lambda(r)$. From (2.1) we get

$$
|\theta(r)| \cdot\left\|\varphi_{r}\right\|_{\infty} \leq M, \quad r_{0}<r<1 .
$$

Using the property 2$)$ of the kernels $\left\{\varphi_{r}(t)\right\}$ we may define a collection of numbers $\varepsilon_{r}>0$ such that

$$
\varepsilon_{r} \searrow 0, \quad \delta_{r}=\sup \left\{|t|: \varphi_{r}(t) \geq \varepsilon_{r}\right\} \rightarrow 0 \text { as } r \rightarrow 1 .
$$

Applying Lemma 3.3 for any $0<r<1$ we define a family of intervals $\left\{I_{j}^{(r)}, j=\right.$ $\left.1,2, \ldots, n_{r}\right\}$ such that

$$
\begin{gathered}
\left|I_{j}^{(r)}\right| \leq 2 \delta_{r}, \quad j=1,2, \ldots, n_{r}, \\
\sum_{j=1}^{n_{r}}\left|I_{j}^{(r)}\right|<10\left(\varepsilon_{r}\right)^{-1} \max \left\{1,|\theta(r)| \cdot\left\|\varphi_{r}\right\|_{\infty},\left\|\varphi_{r}\right\|_{1}\right\}, \\
\left|\varphi_{r}(\theta(r)-t)-\sum_{j=1}^{n_{r}} \varepsilon_{j}^{(r)} \mathbb{I}_{I_{j}^{(r)}}(t)\right| \leq \varepsilon_{r},
\end{gathered}
$$

where $\varepsilon_{j}^{(r)}= \pm \varepsilon_{r}$. From (2.11) and (2.14) we conclude

$$
\varepsilon_{r} \cdot \sum_{j=1}^{n_{r}}\left|I_{j}^{(r)}\right| \leq L, \quad r_{0}<r<1,
$$

where $L$ is a positive constant. From (2.12) and (2.15) we obtain

$$
\Phi_{r}(\theta(r), d \mu)=\int_{\mathbb{T}} \varphi_{r}(\theta(r)-t) d \mu(t)=\sum_{j=1}^{n_{r}} \varepsilon_{j}^{(r)} \int_{I_{j}^{(r)}} d \mu(t)+o(1),
$$

where $o(1) \rightarrow 0$ as $r \rightarrow 1$. Using this, we get

$$
\left|\Phi_{r}(\theta(r), d \mu)\right| \leq \varepsilon_{r} \cdot \sum_{j=1}^{n_{r}}\left|I_{j}^{(r)}\right| \cdot \frac{1}{\left|I_{j}^{(r)}\right|}\left|\int_{I_{j}^{(r)}} d \mu(t)\right|+o(1) .
$$


According to (2.12) and (2.13), we have

$$
\max _{1 \leq j \leq n_{r}} \frac{1}{\left|I_{j}^{(r)}\right|}\left|\int_{I_{j}^{(r)}} d \mu(t)\right| \rightarrow \mu^{\prime}(0)=0 \text { as } r \rightarrow 1 .
$$

This together with (2.16) and (2.17) implies that $\Phi_{r}(\theta(r), d \mu) \rightarrow 0$ as $r \rightarrow 1$.

Proof of Theorem 2.2. Let $\theta(r)$ satisfies (2.11). We again assume that $x_{0}=0$, $\mu^{\prime}\left(x_{0}\right)=0$ and so we will have $|\mu|^{\prime}(0)=0$. Then, repeating the same process of the proof of Theorem 2.1 at this time for the functions $\varphi_{r}^{*}(t)$ together with the measure $|\mu|$, instead of (2.17) we obtain

$$
\int_{\mathbb{T}} \varphi_{r}^{*}(\theta(r)-t) d|\mu|(t)=\sum_{j=1}^{n_{r}} \varepsilon_{j}^{(r)} \int_{I_{j}^{(r)}} d|\mu|(t)+o(1) .
$$

Then we get

$$
\begin{aligned}
\left|\Phi_{r}(\theta(r), d \mu)\right| & \leq \int_{\mathbb{T}} \varphi_{r}^{*}(\theta(r)-t) d|\mu|(t) \\
& =\varepsilon_{r} \cdot \sum_{j=1}^{n_{r}}\left|I_{j}^{(r)}\right| \cdot \frac{1}{\left|I_{j}^{(r)}\right|} \int_{I_{j}^{(r)}} d|\mu|(t)+o(1) .
\end{aligned}
$$

Since $|\mu|(t)$ is differentiable at 0 , we get

$$
\Phi_{r}(\theta(r), d \mu) \rightarrow 0 .
$$

Proof of Theorem 2.3. For any $0<r<1$ there exist a point $x_{r} \in(0, \pi)$, a number $0<\delta_{r}<\lambda(r) / 4$ and a measurable set $E_{r}$ such that

$$
\begin{array}{r}
E_{r} \subset\left(x_{r}-\delta_{r}, x_{r}+\delta_{r}\right), \quad\left|E_{r}\right|>\frac{3 \delta_{r}}{2}, \\
\varphi_{r}(x)>\frac{\left\|\varphi_{r}\right\|_{\infty}}{2}, \quad x \in E_{r} .
\end{array}
$$

From these relations it follows that $\varphi_{r}^{*}(x)>\left\|\varphi_{r}\right\|_{\infty} / 2$ if $x \in\left(0,\left|x_{r}\right|\right)$. On the other hand, by property 3$)$ we have $\left\|\varphi_{r}^{*}\right\|_{1} \leq M$ for some constant $M>0$. Thus we get

$$
\left|x_{r}\right| \leq \frac{2 M}{\left\|\varphi_{r}\right\|_{\infty}}, \quad 0<r<1
$$

Denote

$$
\begin{gathered}
n(r)=\left[\frac{4 \pi}{\lambda(r)}\right] \in \mathbb{N}, \\
\Delta_{r}=\bigcup_{k=0}^{n(r)-1}\left[\frac{2 \pi k}{n(r)}-\delta_{r}, \frac{2 \pi k}{n(r)}+\delta_{r}\right],
\end{gathered}
$$

and consider the function

$$
f_{r}(x)=\frac{\mathbb{I}_{\Delta_{r}(x)}}{\left|\Delta_{r}\right|}=\frac{\mathbb{I}_{\Delta_{r}}(x)}{2 \delta_{r} n(r)} .
$$

If $x \in \mathbb{T}$ is an arbitrary point, then

$$
x \in\left[\frac{2 \pi k_{0}}{n(r)}, \frac{2 \pi\left(k_{0}+1\right)}{n(r)}\right)
$$


for some $k_{0}, 0 \leq k_{0}<n(r)$. Taking $\theta=x-x_{r}-2 \pi k_{0} / n(r)$, from (2.20) and (2.21) we obtain

$$
|\theta|<\frac{2 \pi}{n(r)}+\left|x_{r}\right|<\frac{2 \pi \lambda(r)}{4 \pi-\lambda(r)}+\frac{2 M}{\left\|\varphi_{r}\right\|_{\infty}}=\lambda(r)\left(\frac{1}{2}+\frac{2 M}{\lambda(r)\left\|\varphi_{r}\right\|_{\infty}}\right) .
$$

Using (2.2), we may fix a sequence $r_{k} \nearrow 1$ such that

$$
\lambda\left(r_{k}\right)\left\|\varphi_{r_{k}}\right\|_{\infty}>M \cdot 4^{k}, \quad k=1,2, \ldots
$$

From (2.23) and (2.24) we conclude

$$
|\theta|<\lambda(r), \text { if } r=r_{k}
$$

Since $\varphi_{r}(x) \geq 0$, using (2.19) and (2.21), for the same $x$ we get

$$
\begin{aligned}
\Phi_{r}\left(x-\theta, f_{r}\right) & \\
& =\int_{\mathbb{T}} \varphi_{r}\left(2 \pi k_{0} / n(r)+x_{r}-t\right) f_{r}(t) d t \\
& \geq \frac{1}{2 \delta_{r} n(r)} \int_{2 \pi k_{0} / n(r)-\delta_{r}}^{2 \pi k_{0} / n(r)+\delta_{r}} \varphi_{r}\left(2 \pi k_{0} / n(r)+x_{r}-t\right) d t \\
& =\frac{1}{2 \delta_{r} n(r)} \int_{x_{r}-\delta_{r}}^{x_{r}+\delta_{r}} \varphi_{r}(u) d u \\
& \geq \frac{1}{2 \delta_{r} n(r)} \cdot \frac{3 \delta_{r}}{2} \cdot \frac{\left\|\varphi_{r}\right\|_{\infty}}{2} \geq \frac{3 \lambda(r)\left\|\varphi_{r}\right\|_{\infty}}{16} .
\end{aligned}
$$

From (2.24), (2.25) and (2.26) we obtain

$$
\sup _{\theta \in \lambda\left(r_{k}, x\right)} \Phi_{r_{k}}\left(\theta, f_{r_{k}}\right) \geq 3 M \cdot 4^{k-2}, \quad x \in \mathbb{T} .
$$

Using (2.24), we define

$$
f(x)=\sum_{k=1}^{\infty} 2^{-k} f_{r_{k}}(x) \in L^{1}(\mathbb{T})
$$

From (2.27) and (2.24) it follows that

$$
\sup _{\theta \in \lambda\left(r_{k}, x\right)} \Phi_{r_{k}}(\theta, f) \geq \sup _{\theta \in \lambda\left(r_{k}, x\right)} 2^{-k} \Phi_{r_{k}}\left(\theta, f_{r_{k}}\right) \geq 3 M \cdot 2^{k-4},
$$

which implies (2.3).

\section{Fatou type theorems: The CASE $L^{\infty}$}

Let $\lambda(r):(0,1) \rightarrow \mathbb{R}$ be an arbitrary real function with $\lambda(r) \rightarrow 0$ as $r \rightarrow 1$. The quantity

$$
\gamma_{\lambda}=\lim _{\tau \rightarrow 1}\left(\limsup _{\delta \rightarrow 0} \sup _{\tau<r<1} \int_{-\delta \lambda(r)}^{\delta \lambda(r)} \varphi_{r}(t) d t\right)
$$

completely characterizes the almost everywhere $\lambda(r)$ convergence property of $\Phi_{r}(x, f)$ in $L^{\infty}(\mathbb{T})$.

Theorem 3.1. If $\left\{\varphi_{r}(x)\right\}$ is a regular AI consisting of even functions and $\gamma_{\lambda}=0$, then for any $f \in L^{\infty}(\mathbb{T})$ the relation

$$
\lim _{\substack{r \rightarrow 1 \\ \theta \in \lambda(r, x)}} \Phi_{r}(\theta, f)=f(x)
$$


holds at any Lebesgue point.

Theorem 3.2. If $\left\{\varphi_{r}(x)\right\}$ is a regular AI consisting of even functions and $\gamma_{\lambda}>0$, then there exists a set $E \subset \mathbb{T}$, such that $\Phi_{r}\left(x, \mathbb{I}_{E}\right)$ is $\lambda(r)$-divergent at any $x \in \mathbb{T}$.

Note that if $\lambda(t)$ satisfies the condition (2.1), then $\gamma_{\lambda}=0$. One can easily construct a family of kernels $\left\{\varphi_{r}\right\}$ such that $\gamma_{\lambda}=0$, but (2.1) is not satisfied. This means the $\gamma_{\lambda}=0$ is a weaker condition than (2.1).

Proof of Theorem 3.1. Since $\gamma_{\lambda}=0$ for any $0<\varepsilon<1 / 2$ we may chose $\delta>0$ and $0<\tau<1$, such that

$$
\int_{-\delta \lambda(r)}^{\delta \lambda(r)} \varphi_{r}(t) d t<\varepsilon, \quad \tau<r<1
$$

Then we define

$$
\varphi_{r}^{(1)}(x)=\left\{\begin{array}{lll}
\varphi_{r}(x)-\varphi_{r}(\delta \lambda(r)) & \text { if } \quad|x| \leq \delta \lambda(r) \\
0 & \text { if } \quad \delta \lambda(r)<|x|<\pi .
\end{array}\right.
$$

and

where

$$
\varphi_{r}^{(2)}(x)=\frac{\varphi_{r}(x)-\varphi_{r}^{(1)}(x)}{\left\|\varphi_{r}(x)-\varphi_{r}^{(1)}\right\|_{L^{1}}}=\frac{\varphi_{r}(x)-\varphi_{r}^{(1)}(x)}{1-l_{r}}
$$

$$
l_{r}=\int_{-\delta \lambda(r)}^{\delta \lambda(r)}\left(\varphi_{r}(t)-\varphi_{r}(\delta \lambda(r)) d t<\varepsilon<\frac{1}{2}, \quad \tau<r<1 .\right.
$$

It is clear, that $\left\{\varphi_{r}^{(2)}\right\}$ is a regular AI and we have

$$
\varphi_{r}(x)=\varphi_{r}^{(1)}(x)+\left(1-l_{r}\right) \varphi_{r}^{(2)}(x) .
$$

From (3.1) it follows that

$$
\left|\int_{\mathbb{T}} \varphi_{r}^{(1)}(x-t) f(t) d t\right| \leq\|f\|_{\infty} \int_{-\delta \lambda(r)}^{\delta \lambda(r)} \varphi_{r}(t) d t \leq \varepsilon\|f\|_{\infty}
$$

and

$$
\varphi_{r}(\delta \lambda(r)) \cdot 2 \delta \lambda(r)<\varepsilon, \quad \tau<r<1 .
$$

Thus, using the definition of $\varphi_{r}^{(2)}(x)$, we get

$$
\left\|\varphi_{r}^{(2)}\right\|_{\infty} \cdot \lambda(r)<\frac{\varepsilon}{2 \delta\left(1-l_{r}\right)}<\frac{\varepsilon}{4 \delta}
$$

Using this and Theorem 2.1 we conclude, that

$$
\lim _{\substack{r \rightarrow 1 \\ \theta \in \lambda(r, x)}} \int_{\mathbb{T}} \varphi_{r}^{(2)}(\theta-t) f(t) d t=f(x)
$$

at any Lebesgue point. Now without loss of generality we assume that $f(x) \geq 0$. If $x$ is an arbitrary Lebesgue point, using (3.2), (3.3) and (3.4) we get

$$
\begin{aligned}
& \limsup _{\substack{r \rightarrow 1 \\
\theta \in \lambda(r, x)}} \Phi_{r}(\theta, f) \leq \varepsilon\|f\|_{\infty}+f(x), \\
& \liminf _{\substack{r \rightarrow 1 \\
\theta \in \lambda(r, x)}} \Phi_{r}(\theta, f) \geq-\varepsilon\|f\|_{\infty}+(1-\varepsilon) f(x) .
\end{aligned}
$$


Since $\varepsilon$ can be taken sufficiently small, we get

$$
\lim _{\substack{r \rightarrow 1 \\ \theta \in \lambda(r, x)}} \Phi_{r}(\theta, f)=f(x)
$$

and the theorem is proved.

If $f(x)$ is a function defined on a set $E \subset \mathbb{T}$ we denote

$$
\mathrm{OSC}_{x \in E} f(x)=\sup _{x, y \in E}|f(x)-f(y)|
$$

Lemma 3.3. Let

$$
U_{n}^{\delta}=\bigcup_{k=0}^{n-1}\left(\frac{\pi(2 k+1-\delta)}{n}, \frac{\pi(2 k+1+\delta)}{n}\right), \quad n \in \mathbb{N}, \quad 0<\delta<\frac{1}{2},
$$

and $J \subset \mathbb{T}, \pi>|J| \geq 16 \pi / n$, is an arbitrary closed interval. If a measurable set $E \subset \mathbb{T}$ satisfies either

$$
E \cap J=J \cap U_{n}^{\delta} \text { or } E \cap J=J \backslash U_{n}^{\delta}
$$

and $\varphi(x) \in L^{\infty}(\mathbb{T})$ is an even decreasing on $[0, \pi]$ function, then

$$
\operatorname{OSC}_{\theta \in\left[x-\frac{4 \pi}{n}, x+\frac{4 \pi}{n}\right]} \int_{\mathbb{T}} \varphi(\theta-t) \mathbb{I}_{E}(t) d t>\int_{-\frac{\pi \delta}{n}}^{\frac{\pi \delta}{n}} \varphi(t) d t-16 \delta-2 \pi \varphi\left(\frac{|J|}{4}\right)
$$

for any $x \in J$.

Proof. We suppose $J=[a, b]$ and

$$
\frac{2 \pi(p-1)}{n}<a \leq \frac{2 \pi p}{n}, \quad \frac{2 \pi(q-1)}{n}<b \leq \frac{2 \pi q}{n} .
$$

First we consider the case

$$
E \cap J=J \cap U_{n}^{\delta}
$$

If $x \in J$, then

$$
x \in I=\left[\frac{2 \pi(m-1)}{n}, \frac{2 \pi m}{n}\right]
$$

for some $p \leq m \leq q$. Without loss of generality we may assume that the center of $I$ is on the left hand side of the center of $J$. Then we will have

$$
b-\frac{2 \pi(m+1)}{n} \geq \frac{|J|}{2}-\frac{4 \pi}{n} \geq \frac{|J|}{4} .
$$

It is clear, that the points

$$
\theta_{1}=\frac{2 \pi m}{n}+\frac{\pi}{n}, \quad \theta_{2}=\frac{2 \pi(m+1)}{n}
$$


are in the interval $[0, x+4 \pi / n]$. Besides we have

$$
\begin{aligned}
\int_{\mathbb{T}} \varphi\left(\theta_{1}-t\right) \mathbb{I}_{E}(t) d t & -\int_{\mathbb{T}} \varphi\left(\theta_{2}-t\right) \mathbb{I}_{E}(t) d t \\
& =\int_{\theta_{2}-\pi}^{a}\left[\varphi\left(\theta_{1}-t\right)-\varphi\left(\theta_{2}-t\right)\right] \mathbb{I}_{E}(t) d t \\
& +\int_{a}^{b}\left[\varphi\left(\theta_{1}-t\right)-\varphi\left(\theta_{2}-t\right)\right] \mathbb{I}_{E}(t) d t \\
& +\int_{b}^{\theta_{2}+\pi}\left[\varphi\left(\theta_{1}-t\right)-\varphi\left(\theta_{2}-t\right)\right] \mathbb{I}_{E}(t) d t \\
& =A_{1}+A_{2}+A_{3} .
\end{aligned}
$$

Since $\varphi$ is decreasing on $[0, \pi]$ we have

$$
A_{1} \geq 0 \text {. }
$$

If $t \in\left[b, \theta_{2}+\pi\right]$ then, using (3.7), we get

$$
t-\theta_{2} \geq b-\theta_{2} \geq \frac{|J|}{4}, \quad t-\theta_{1} \geq \frac{|J|}{4},
$$

which implies

$$
\left|A_{3}\right| \leq 2 \pi \varphi\left(\frac{|J|}{4}\right)
$$

To estimate $A_{2}$ we denote

$$
a_{k}=\int_{\pi(k-\delta) / n}^{\pi(k+\delta) / n} \varphi(t) d t, \quad k \in \mathbb{Z} .
$$

We have

$$
a_{0}=\int_{-\pi \delta / n}^{\pi \delta / n} \varphi(t) d t
$$

Using properties of $\varphi$ we have $a_{k}=a_{-k}$ and $a_{1} \geq a_{2} \geq \ldots$ Using Chebishev inequality we have $\varphi(t) \leq 1 / t$. Thus we obtain

$$
a_{k} \leq a_{1}=\int_{\pi(1-\delta) / n}^{\pi(1+\delta) / n} \varphi(t) d t \leq \frac{2 \pi \delta / n}{\pi(1-\delta) / n}=\frac{2 \delta}{1-\delta}<4 \delta, \quad k \geq 1 .
$$

Using (3.6), (3.11), (3.12) and (3.13), we get

$$
\begin{aligned}
A_{2} & \geq \sum_{k=p}^{q-2} \int_{\pi(2 k+1-\delta) / n}^{\pi(2 k+1+\delta) / n}\left[\varphi\left(\theta_{1}-t\right)-\varphi\left(\theta_{2}-t\right)\right] d t-8 \delta \\
& =\sum_{k=p}^{q-1} \int_{\pi(2(m-k)-\delta) / n}^{\pi(2(m-k)+\delta) / n} \varphi(t) d t-\sum_{k=p}^{q-1} \int_{\pi(2(m-k)+1-\delta) / n}^{\pi(2(m-k)+1+\delta) / n} \varphi(t) d t-8 \delta \\
& =\sum_{k=m-q+1}^{m-p} a_{2 k}-\sum_{k=m-q+1}^{m-p} a_{2 k+1}-8 \delta \geq a_{0}-a_{1}-a_{-1}-8 \delta \\
& >\int_{-\pi \delta / n}^{\pi \delta / n} \varphi(t) d t-16 \delta .
\end{aligned}
$$


Combining this with (3.9) and (3.10), we get

$$
\int_{\mathbb{T}} \varphi\left(\theta_{1}-t\right) \mathbb{I}_{E}(t) d t-\int_{\mathbb{T}} \varphi\left(\theta_{2}-t\right) \mathbb{I}_{E}(t) d t \geq \int_{-\frac{\pi \delta}{n}}^{\frac{\pi \delta}{n}} \varphi(t) d t-16 \delta-2 \pi \varphi\left(\frac{|J|}{4}\right),
$$

which together with (3.8) implies (3.5). To deduce the case $E \cap J=J \backslash U_{n}^{\delta}$ notice, that for the complement $E^{c}$ we have $E^{c} \cap J=J \cap U_{n}^{\delta}$ and so (3.5) holds for $E^{c}$. Therefore we obtain

$$
\begin{aligned}
\operatorname{OSC}_{\theta \in\left[x-\frac{4 \pi}{n}, x+\frac{4 \pi}{n}\right]} & \int_{\mathbb{T}} \varphi(\theta-t) \mathbb{I}_{E}(t) d t \\
& =\operatorname{OSC}_{\theta \in\left[x-\frac{4 \pi}{n}, x+\frac{4 \pi}{n}\right]}\left(\|\varphi\|_{1}-\int_{\mathbb{T}} \varphi(\theta-t) \mathbb{I}_{E}(t) d t\right) \\
& =\operatorname{OSC}_{\theta \in\left[x-\frac{4 \pi}{n}, x+\frac{4 \pi}{n}\right]}\left(\int_{\mathbb{T}} \varphi(\theta-t) \mathbb{I}_{E^{c}}(t) d t\right) \\
& >\int_{-\frac{\pi \delta}{n}}^{\frac{\pi \delta}{n}} \varphi(t) d t-16 \delta-2 \pi \varphi\left(\frac{|J|}{4}\right),
\end{aligned}
$$

which completes the proof of the lemma.

Proof of Theorem 3.2. Since $\gamma_{\lambda}>0$, there exist sequences $\delta_{k} \searrow 0$ and $r_{k} \rightarrow 1$, such that

$$
\int_{-\delta_{k} \lambda\left(r_{k}\right)}^{\delta_{k} \lambda\left(r_{k}\right)} \varphi_{r_{k}}(t) d t>\frac{\gamma_{\lambda}}{2}, \quad k=1,2, \ldots
$$

Denote

$$
U_{k}=U_{n_{k}}^{\delta_{k}}, \quad n_{k}=\left[\frac{\pi}{\lambda\left(r_{k}\right)}\right],
$$

where $U_{n}^{\delta}$ is defined in the Lemma 3.3. Define the sequences of measurable sets $E_{n}$ by

$$
E_{1}=U_{1}, \quad E_{k}=E_{k-1} \triangle U_{k}=\left(E_{k-1} \backslash U_{k}\right) \cup\left(U_{k} \backslash E_{k-1}\right), \quad k>1
$$

We say $J$ is an adjacent interval for $E_{k}$, if it is a maximal interval containing either in $E_{k}$ or $\left(E_{k}\right)^{c}$. The family of all this intervals form a covering of whole $\mathbb{T}$. It is easy to observe, that a suitable selection of $\delta_{k}$ and $r_{k}$ may provide

$$
\begin{aligned}
& \varphi_{r_{k}}\left(\frac{|J|}{4}\right)<\frac{\gamma_{\lambda}}{16 \pi}, \text { if } J \text { is adjacent for } E_{k-1}, \\
& \delta_{j} \leq \frac{\gamma_{\lambda}}{2^{j+5}\left\|\varphi_{r_{k}}\right\|_{\infty}}, \quad j \geq k+1,
\end{aligned}
$$

It is easy to observe, that if $k<m$, then

$$
\left\|\mathbb{I}_{E_{k}}-\mathbb{I}_{E_{m}}\right\|_{1}=\left|E_{k} \triangle E_{m}\right| \leq \sum_{j \geq k+1}\left|U_{j}\right|
$$

This implies, that $\mathbb{I}_{E_{n}}(t)$ converges to a function $f(t)$ in $L^{1}$. Using Egorov's theorem, we conclude that $f(t)=\mathbb{I}_{E}(t)$ for a measurable set $E \subset \mathbb{T}$. Tending $m$ to infinity, from (3.17) and (3.18) we get

$$
\left|E_{k} \triangle E\right| \leq\left|\bigcup_{j \geq k+1} U_{j}\right| \leq 2 \pi \sum_{j \geq k+1} \delta_{j} \leq \frac{\gamma_{\lambda}}{16\left\|\varphi_{r_{k}}\right\|_{\infty}} .
$$


Fix a point $x \in \mathbb{T}$. We have $x \in J$ where $J$ is an adjacent interval for $E_{k-1}$. From the definition of $E_{k}$ it follows that either

$$
E_{k} \cap J=J \cap U_{k} \text { or } E_{k} \cap J=J \backslash U_{k} .
$$

From (3.15) we have

$$
\lambda\left(r_{k}, x\right)=\left(x-\lambda\left(r_{k}\right), x+\lambda\left(r_{k}\right)\right) \subset\left[x-\frac{4 \pi}{n_{k}}, x+\frac{4 \pi}{n_{k}}\right] .
$$

Thus, applying Lemma 3.3, (3.14) and (3.16), we get

$$
\begin{aligned}
\operatorname{OSC}_{\theta \in \lambda\left(r_{k}, x\right)} & \Phi_{r_{k}}\left(\theta, \mathbb{I}_{E_{k}}\right) \\
& \geq \operatorname{OSC}_{\theta \in\left[x-\frac{4 \pi}{n_{k}}, x+\frac{4 \pi}{n_{k}}\right]} \Phi_{r_{k}}\left(\theta, \mathbb{I}_{E_{k}}\right) \\
& \geq \int_{-\frac{\pi \delta_{k}}{n_{k}}}^{\frac{\pi \delta_{k}}{n_{k}}} \varphi_{r_{k}}(t) d t-16 \delta_{k}-2 \pi \varphi_{r_{k}}\left(\frac{|J|}{4}\right) \\
& \geq \int_{-\delta_{k} \lambda\left(r_{k}\right)}^{\delta_{k} \lambda\left(r_{k}\right)} \varphi_{r_{k}}(t) d t-16 \delta_{k}-\frac{\gamma_{\lambda}}{8} \\
& \geq \frac{\gamma_{\lambda}}{4}-16 \delta_{k} .
\end{aligned}
$$

From (3.19) we conclude

$$
\begin{aligned}
\operatorname{OSC}_{\theta \in \lambda\left(r_{k}, x\right)} & \Phi_{r_{k}}\left(\theta, \mathbb{I}_{E}\right) \\
& >\operatorname{OSC}_{\theta \in \lambda\left(r_{k}, x\right)} \Phi_{r_{k}}\left(\theta, \mathbb{I}_{E_{k}}\right)-\frac{\gamma_{\lambda}}{16} \geq \frac{\gamma_{\lambda}}{8}-16 \delta_{k},
\end{aligned}
$$

which completes the proof of theorem since $\delta_{k} \rightarrow 0$.

\section{FinAl REMARKS}

In the definition of $\lambda(r)$-convergence the range of the parameter $r$ is $(0,1)$ with the limit point 1 . Certainly it is not essential in the theorems. We could take any set $Q \in \mathbb{R}$ with limit point $r_{0}$ which is either a finite number or $\infty$. We may define an approximation of the identity on the real line to be a family of functions $\varphi_{r} \in L^{\infty}(\mathbb{R}) \cap L^{1}(\mathbb{R}), r>0$, satisfying the same conditions 1)-3) as AI on $\mathbb{T}$ has. We have just make a little change in the condition 2), that is $\left\|\varphi_{r}^{*} \cdot \mathbb{I}_{\{|t| \geq \delta\}}\right\|_{1} \rightarrow 0$ as $r \rightarrow 0, \delta>0$. In this case usually convergence is considered while $r \rightarrow 0$. Analogous results can be formulated and proved for the integrals

$$
\Phi_{r}(x, d \mu)=\int_{\mathbb{R}} \varphi_{r}(t-x) d \mu(t), \quad r>0 .
$$

And it can be done just repeating the above proofs after miserable changes.

Any function $\Phi(x) \in L^{\infty}(\mathbb{R}) \cap L^{1}(\mathbb{R})$ defines an approximation of identity by $\varphi_{r}(x)=r \Phi(x / r)$ as $r \rightarrow 0$. The operators corresponding to such kernels in higher dimensional case investigated by E. M. Stain ([17, p. 57). We note for such kernels we have $\left\|\varphi_{r}\right\|_{\infty}=r^{-1}\|\Phi\|_{\infty}$ and therefore the condition (2.1) takes the form $\lambda(r) \leq c r$. This bound characterizes the nontangential convergence in the upper half plane and it turns out to be a necessary and sufficient condition for almost everywhere $\lambda(r)$-convergence of the integrals (4.1). 
P. Sjögren ([14, 15], 16]), J.-O. Rönning (10, 11], 12]), I. N. Katkovskaya and V. G. Krotov (6] ) considered the square root Poisson integrals

$$
\mathcal{P}_{r}^{0}(x, f)=\frac{1}{c(r)} \int_{\mathbb{T}}\left[P_{r}(x-t)\right]^{1 / 2} f(t) d t
$$

where

$$
c(r)=\int_{\mathbb{T}}\left[P_{r}(t)\right]^{1 / 2} d t
$$

is the normalizing coefficient. They proved, that

$$
\lim _{r \rightarrow 1} \mathcal{P}_{r}^{0}(x+\theta(r), f)=f(x) \text { a.e. }
$$

whenever $f \in L^{p}(\mathbb{T}), 1 \leq p \leq \infty$, and

$$
|\theta(r)| \leq\left\{\begin{array}{lll}
c(1-r)\left(\log \frac{1}{1-r}\right)^{p} & \text { if } & 1 \leq p<\infty, \\
(1-r)^{1-\varepsilon}, & \text { if } & p=\infty .
\end{array}\right.
$$

The case of $p=1$ is proved in [14, $1<p \leq \infty$ is considered in [10, 11]. They provide also some weak type inequalities for the maximal operators of square root Poisson integrals. In the paper ([6]) authors obtained weighted strong type inequalities for the same operators. The cases $p=1$ and $p=\infty$ are consequences of the Theorem 2.1 with an additional information about the points where the convergence occurs.

At the end of the paper we would like to bring couple of consequences of our theorems, that we consider interesting.

Corollary 4.1. If $\sigma_{n}(x, f)$ are the Fejer means of Fourier series of a function $f \in L^{1}(\mathbb{T})$ and $\theta_{n}=O(1 / n)$, then $\sigma_{n}\left(x+\theta_{n}, f\right) \rightarrow f(x)$ at any Lebesgue point $x \in \mathbb{T}$.

Corollary 4.2. If $S_{n}(x, f)$ are the partial sums of a function $f \in L^{1}(0,1)$ in Franklin system and $\theta_{n}=O(1 / n)$, then $S_{n}\left(x+\theta_{n}, f\right) \rightarrow f(x)$ at any Lebesgue point $x \in \mathbb{T}$.

\section{REFERENCES}

[1] H. Aikawa, Harmonic functions having no tangential limits, Proc. Amer. Math. Soc., 1990, vol. 108 , no. $2,457-464$.

[2] H. Aikawa, Harmonic functions and Green potential having no tangential limits, J. London Math. Soc., 1991, vol. 43, 125-136.

[3] F. Di Biase, Tangential curves and Fatou's theorem on trees, J. London Math. Soc., 1998, vol. 58 , no. $2,331-341$.

[4] F. Di Biase, A. Stokolos, O. Svensson and T. Weiss, On the sharpness of the Stolz approach, Annales Acad. Sci. Fennicae, 2006, vol. 31, 47-59.

[5] P. Fatou, Séries trigonométriques et séries de Taylor, Acta Math., 1906, vol. 30, 335-400.

[6] I. N. Katkovskaya and V. G. Krotov, Strong-Type Inequality for Convolution with Square Root of the Poisson Kernel, Mathematical Notes, 2004, vol. 75, no. 4, 542552.

[7] J. E. Littlewood, On a theorem of Fatou, Journal of London Math. Soc., 1927, vol. 2, 172-176.

[8] A. J. Lohwater and G. Piranian, The boundary behavior of functions analytic in unit disk, Ann. Acad. Sci. Fenn., Ser A1, 1957, vol. 239, 1-17.

[9] A. Nagel and E. M. Stein, On certain maximal functions and approach regions, Adv. Math., 1984, vol. 54, 83-106.

[10] J.-O. Rönning, Convergence results for the square root of the Poisson kernel, Math. Scand., 1997, vol. 81, no. 2, 219-235.

[11] J.-O. Rönning, On convergence for the square root of the Poisson kernel in symmetric spaces of rank 1, Studia Math., 1997, vol. 125, no. 3, 219-229. 
[12] J.-O. Rönning, Convergence results for the square root of the Poisson kernel in the bidisk, Math. Scand., 1999, vol. 84, no. 1, 81-92.

[13] S. Saeki, On Fatou-type theorems for non radial kernels, Math. Scand., 1996, vol. 78, 133-160.

[14] P. Sjog̈ren, Une remarque sur la convergence des fonctions propres du laplacien à valeur propre critique, Théorie du potentiel (Orsay, 1983), Lecture Notes in Math., vol. 1096, Springer, Berlin, 1984, pp. 544-548

[15] P. Sjog̈ren, Convergence for the square root of the Poisson kernel, Pacific J. Math., 1988, vol. 131, no 2, 361-391.

[16] P. Sjog̈ren, Approach regions for the square root of the Poisson kernel and bounded functions, Bull. Austral. Math. Soc., 1997, vol. 55, no 3, 521-527.

[17] E. M. Stein, Harmonic Analysis, Princeton University Press, 1993.

Institute of Mathematics of Armenian National Academy of Sciences, Baghramian Ave.- 24/5, 0019, Yerevan, Armenia

E-mail address: g.karagulyan@yahoo.com

Yerevan State University, Alek Manukyan, 1, 0049, Yerevan, Armenia

E-mail address: mher.safaryan@gmail.com 